\title{
The universality hypothesis: binary and stellar populations in star clusters and galaxies
}

\author{
Pavel Kroupa \\ Argelander-Institut für Astronomie, Universität Bonn, Auf dem Hügel 71, D-53121 Bonn, \\ Germany \\ email: pavel@astro.uni-bonn.de
}

\begin{abstract}
It is possible to extract, from the observations, distribution functions of the birth dynamical properties of a stellar population, and to also infer that these are quite invariant to the physical conditions of star formation. The most famous example is the stellar IMF, and the initial binary population (IBP) seems to follow suit. A compact mathematical formulation of the IBP can be derived from the data. It has three broad parts: the IBP of the dominant stellar population $\left(0.08-2 M_{\odot}\right)$, the IBP of the more-massive stars and the IBP of brown dwarfs. These three mass regimes correspond to different physical regimes of star formation but not to structure in the IMF. With this formulation of the IBP it becomes possible to synthesize the stellar-population of whole galaxies.
\end{abstract}

Keywords. stars: formation; stars: low-mass, brown dwarfs; stars: early-type; stars: pre-mainsequence; stars: luminosity function, mass function; binaries: general; open clusters and associations: general; galaxies: star clusters; galaxies: stellar content; methods: n-body simulations,

\section{Introduction}

The fundamental dynamical properties of stellar populations are the masses of the stars and their correlation in multiple stellar systems. The distribution of stellar masses at birth, the IMF, is rather well constrained and has (surprisingly) been found to be invariant despite theoretical models predicting systematic variation for example of the mean stellar mass or even of the minimum mass with the physical conditions of star formation. This problematical issue has been discussed at some length by Kroupa (2008), where the IMF UNIVERSALITY HyPOTHESIS is stated.

Equivalently, the question may be raised whether the other distribution functions characterizing a stellar population, namely the distribution functions of binary systems, are just as invariant. If this were the case then it would have important bearings on the theory of star formation as the fragmentation length-scale may then not depend much on the physical conditions of the molecular cloud core. A change in the properties of the binary-star distribution functions with mass scale, if found, would yield important clues to the fragmentation and angular momentum re-distribution processes during star formation.

The three important distribution functions describing the initial binary population (IBP) are the distribution of periods $(P$, here always in days), or equivalently of semimajor axes $(a$, in $\mathrm{AU})$, the distribution of mass-ratios $\left(q=m_{2} / m_{1} \leqslant 1\right)$ and the distribution of orbital eccentricities $(e)$. These are related by Kepler's third law: $a^{3} / P_{\mathrm{yr}}^{2}=$ $m_{1}+m_{2}$, where $P_{\mathrm{yr}}$ is the orbital period in years $\left(P=365.25 P_{\mathrm{yr}}\right)$ and $m_{1}, m_{2}$ are the primary- and secondary-star masses in $M_{\odot}$. Because the periods of binary stars range over many orders of magnitude the shorthand $l P \equiv \log _{10} P$ is used throughout this text. 


\section{Star formation and the initial binary population (IBP)}

Observations have shown that the star-formation process is intrinsically linked to the production of binary stars. Indeed, binary stars must be the dominant formation channel because the observed multiplicity fraction (the number of multiple systems divided by the number of sources in the survey) is indistinguishably high among old metal-poor (Carney et al. (2005)) and among thin-disk main-sequence stars (Duquennoy \& Mayor (1991)), and is near unity for pre-main sequence stars and proto-stars (Duchêne (1999), Connelley et al.(2008)). If, on the other hand, higher-order multiple systems were a major outcome of late-type star formation, then the dynamical decay of these on a time-scale $<10^{5} \mathrm{yr}$ would pollute the pre-main-sequence stellar population with single stars which are not observed in large numbers. Indeed, this is evidently the case for massive stars ( $m \gtrsim$ few $M_{\odot}$ ) which appear to form preferentially in binary-rich dense cores of populous embedded clusters which rapidly decay dynamically by ejecting massive stars (Clarke \& Pringle (1992), Pflamm-Altenburg \& Kroupa (2010)). Thus, according to the BinARYStar Conjecture or Theorem (Kroupa (2008)) the vast number of stars form as binaries, while non-hierarchical higher order multiple systems cannot be a significant outcome of late-type star formation.

The formation of binary systems remains an essentially unsolved problem theoretically. Fisher (2004) shows analytically that isolated turbulent cloud cores can produce an unquantifiable fraction of binary systems with the very wide range of orbital periods as observed. But direct cloud collapse calculations are very limited in predicting binary-star properties owing to the severe computational difficulties of treating the magneto-hydrodynamics together with correct radiation transfer and evolving atomic and molecular opacities during collapse.

The currently most advanced hydrodynamical simulations have been reported by Moeckel \& Bate (2010). They allow a turbulent SPH cloud to collapse forming a substantial cluster of 1253 stars and brown dwarfs amounting to $191 M_{\odot}$. The cluster has a half-mass radius of about $0.05 \mathrm{pc}$ and contains a very substantial binary and higher-order multiple stellar population with a large spread of semi-major axes but peaking at a few AU. After dynamical evolution with or without expulsion of the residual gas the distribution of orbits ends up being quite strongly peaked at a few AU with a significant deficit of orbits with $a>10 \mathrm{AU}$, and with a deficit of systems with a mass ratio $q<0.8$, when compared to the main-sequence population (their fig. 11). This state-of-the art computation therewith confirms the above stated issue that it remains a significant challenge for star-formation theory to account for the Gaussian-type distribution of $a$ spanning $10^{-1}-10^{5} \mathrm{AU}$ as for Galactic-field binaries. One essential aspect which is still missing from such computations is stellar feedback which starts heating the cloud as soon as the first proto stars appear. These heating sources are likely to counter the gravitational collapse such that in reality the extreme densities are not achieved allowing a much larger fraction of wide binaries to survive.

More general theoretical considerations suggest that star-formation in dense clusters ought to have a tendency towards a lower binary proportion in warmer molecular clouds (i.e. in cluster-forming cores) because of the reduction of available phase-space for binarystar formation with increasing temperature (Durisen \& Sterzik (1994), hereinafter DS). On the other hand, an enhanced binary proportion for orbital periods $l P \lesssim 5.6$ may be expected in dense clusters due to the stimulation of binary formation through tidal shear (Horton, Bate \& Bonnell (2001)), thus possibly compensating the DS effect. The initial period distribution function (IPF) may thus appear similar in dense and sparse clusters, apart from deviations at long periods due to encounters and the cluster tidal field. 
The multiple-star population in the Galactic field is build-up by star-forming events in star-clusters or groups containing from a dozen to possibly millions of stars. Indeed, a certain but presently not well known fraction of stars form in small- $N$ systems that typically have a size $\approx 100 \mathrm{AU}$, and the dynamical decay of these is likely to affect the final distribution of $P \lesssim 10^{5}$ binaries (Sterzik \& Durisen (1998)), giving rise to non-uniform jet activity (Reipurth (2000)). But again, quantification of their properties is next-toimpossible given the neglect of the hydrodynamical component. But, by the BINARYStar Conjecture above, the binary formation channel must be vastly dominating over the formation of non-hierarchical higher-order multiples.

\section{The IBP universality hypothesis}

It is hoped that star-forming simulations will allow essential insights into reproducing the stellar population stemming from an individual modest star-forming event. But given the computational complexity, synthesizing for example the binary population in a massive star cluster or of a whole galaxy or even parts thereof such as the solar neighborhood are not possible. Therewith it becomes rather apparent that current theory has no predictive power concerning the binary properties of stellar populations in different environments.

However, by proposing that initial distribution functions of the binary-star properties (see end of $\S 1$ ) exist, that is, that there exists an outcome of the isolated (i.e. low-density, such as in Taurus-Auriga) star-formation process that can be quantified in terms of an IBP, we would be put into the situation of being able to synthesize populations. This would become feasible if it is understood how this IBP is affected by physical processes that are inherent to a binary system and that are due to stellar-dynamical encounters in denser star-forming regions.

In fact, the invariance of the IMF must be implying an insensitivity of the starformation outcome to physical conditions. The IMF being invariant constitutes a statistical statement on one of the birth dynamical properties of stars (namely their distribution of masses). So, since both the IMF and the IBP are the result of the same (star-formation) process, and since the IMF is a result of this process "one level deeper down" than the IBP, it is quite natural to suggest that the formal mathematical distribution function of all of the birth dynamical properties of stars are invariant. Thus the following hypothesis follows:

THE STAR-Formation UnIVERSALITY HYPOTHESIS:
IMF universality $\Longleftrightarrow$ IBP universality.

\section{Inferring the IBP for $m<2 M_{\odot}$ stars}

The challenge of inferring the period-, mass-ratio- and eccentricity-distribution functions characterizing the IBP can be formulated as follows: assuming the STAR-Formation UNIVERSALITY HYPOTHESIS to be valid and using observational constraints on the premain sequence binary population, the observed main-sequence Galactic-field binary-star distribution function must be corrected for the dynamical processes acting in the birthgroups or birth-clusters of stars as these emerge into the Galactic field. Fig. 1 visualises this idea.

These dynamical processes are well understood and are detailed in Kroupa (2008): Energy arguments imply the HEGGIE-HILls LAW according to which the wide-binaries are disrupted preferentially compared to short-period binaries and tight binaries typically become tighter. The boundary between the short and long-period binaries depends on 
the velocity dispersion in the birth population. At the same time, binaries with a small mass ratio $($ small $q$ ) are also preferentially disrupted. The tightening of tight binaries implies that such systems and individual stars interacting with them can be ejected from the cluster. Pre-main-sequence EIgEnEvolution evolves the short-period binaries within a time-scale of few $10^{5} \mathrm{yr}$ due to system-internal dissipative processes such as tidalcircularisation, primary-star-disk-secondary-star interactions and disk-disk interactions.

The dynamical disruption of binaries induced through the birth cluster and characterized by the stellar-dynamical operator $\Omega^{M_{\mathrm{ec} 1}, R_{0.5}}$, and the mass-ratio, eccentricity and period evolution induced through pre-main sequence eigenevolution, can both be calculated (see Kroupa (2008) for details). $M_{\text {ecl }}$ is the stellar mass of the embedded cluster with half-mass radius $R_{0.5}$. Note that this "embedded cluster" does not need to be a cluster which survives the first few $10 \mathrm{Myr}$ as a bound star cluster. It can readily be taken to include sparse star-forming aggregates of stars as observed e.g. in the about 0.5 pc radius sub-groups of a dozen pre-main sequence stars in Taurus-Auriga.

The above two-stage transformation of the formal mathematical birth distribution functions $\left(f_{P}, f_{q}, f_{e}\right)$ to the final distribution function (e.g. of the dispersed cluster) can be written as

$$
\mathcal{D}_{\text {outcome }}\left(l P, e, q: m_{1}\right)=\Omega^{M_{\text {ecl }}, R_{0.5}}\left[\Omega_{\text {eigenevol }}\left[\mathcal{D}_{\text {birth }}\left(l P, e, q: m_{1}\right)\right]\right] .
$$

$\mathcal{D}_{\text {birth }}=f_{P}(l P) f_{q}(q) f_{e}(e)$ is the birth distribution function taking the birth period, mass-ratio and eccentricity distribution functions to be separable, that is, the birth parameters $P_{b}, q_{b}, e_{b}$ are not correlated.

A simple theoretical treatment of eigenevolution which is based on pre-main sequence tidal-circularisation theory has been shown to quite nicely account for the correlations between eccentricity, mass-ratio and period for short-period $\left(P \lesssim 10^{3} \mathrm{~d}\right)$ binaries. The

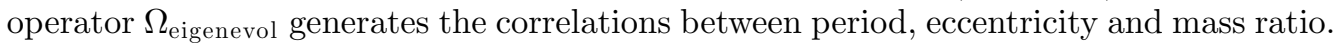

The stellar-dynamical operator, $\Omega^{M_{\mathrm{ec} 1}, R_{0.5}}$, is given by an Nbody star-cluster, and can be envisioned as a transformation of the number of binaries in different binding-energy bins such that the reduction is largest in the most weakly-bound binaries. The tightening of binaries can increase the fraction of short-period binaries in a population.

Putting this together, the birth binary population is defined by the following formal mathematical rules:

THE BIRTH BINARY POPULATION (BBP):

- random pairing from the canonical IMF for $0.08 \lesssim m / M_{\odot} \lesssim 2$;

- thermal eccentricity distribution of eccentricities, $f_{e}(e)=2 e$;

- the period distribution function

$$
f_{P, \text { birth }}=\eta \frac{l P-l P_{\min }}{\delta+\left(l P-l P_{\min }\right)^{2}},
$$

where $\eta=2.5, \delta=45, l P_{\min }=1$ and $\int_{l P_{\min }}^{l P_{\max }} f_{P, \text { birth }} d l P=1$ such that the birth binary fraction is unity $\left(l P_{\max }=8.43\right)$.

Details are provided in Kroupa (2008). Note that customarily the "initial binary population" (IBP) derives from the BBP after pre-main sequence eigenevolution. Given that pre-main sequence eigenevolution occurs mostly for short-period binaries on a time-scale of $10^{5} \mathrm{yr}$ within the system, it is expected to be approximately universal. The STAR Formation Universality Hypothesis thus remains valid. 


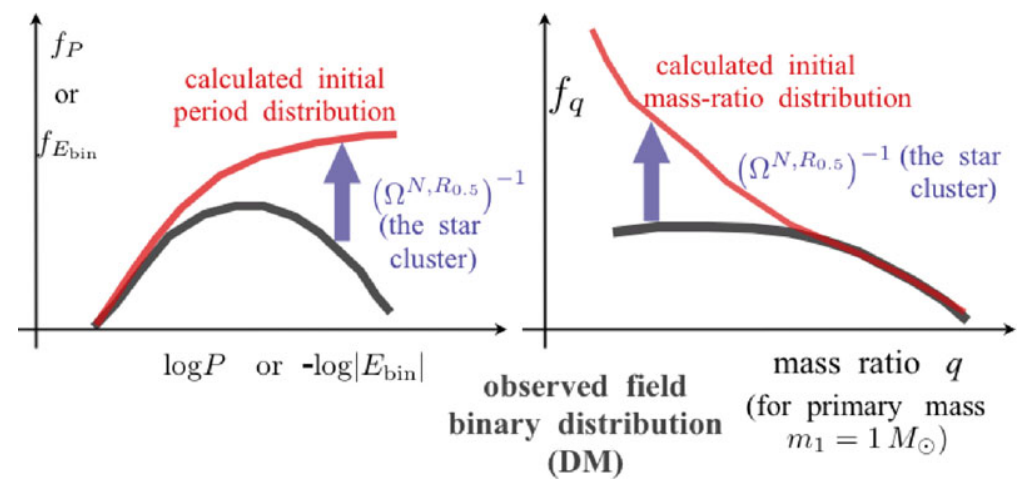

Figure 1. Schematic of how the observed Galactic-field main-sequence (thick black curves, Duquennoy \& Mayor (1991)) period or binding energy distribution (left panel) and the main-sequence mass-ratio distribution (right panel) are back-computed (upwards pointing arrows) with the stellar-dynamical operator $\Omega^{M_{\mathrm{ec} 1}, R_{0.5}}$ to yield an estimate of the initial (thin red curves) period and mass-ratio distributions. Note that the physical reality of $\Omega^{M_{\mathrm{ecl}}, R_{0.5}}$ is established if both $f_{P}$ and $f_{q}$ become consistent with the pre-main sequence data for one and the same $\Omega^{M_{\mathrm{ecl}}, R_{0.5}}$. The eccentricity distribution is not affected by $\Omega^{M_{\mathrm{ecl}}, R_{0.5}}$.

Passing the above easily generated birth distributions through eigenevolution and then letting the stellar-dynamical operator $\Omega^{M_{\mathrm{ecl}}, R_{0.5}}$ act on this resulting initial distribution leads to the Galactic field population as observed. This is shown in Figs $2-3$ for the case $M_{\mathrm{ecl}}=130 M_{\odot}, R_{0.5}=0.8 \mathrm{pc}$, where the arrows indicate the action of $\Omega^{M_{\mathrm{ecl}}, R_{0.5}}$. These results are valid for long-lived cluster models without gas. Short-lived embedded clusters would have a smaller $R_{0.5}$ to give a dynamically-equivalent $\Omega^{M_{\mathrm{ec} 1}, R_{0.5}}$ (Kroupa (1995a)). Note that in Fig. 2 the normalisation of the distribution functions follows the custom

$$
f=\frac{N_{\text {orbits }}}{N_{\text {sys }}},
$$

where $N_{\text {orbits }}$ is the number of binary-star orbits found in a sample of $N_{\text {sys }}=N_{\text {orbits }}+$ $N_{\text {singles }}$ systems or sources, and the $N_{\text {orbits }}$ may be the total number of binaries (for the total binary fraction in a population), or just the number of orbits in an $l P$ interval yielding $f_{P}$.

\section{The IBP for massive stars $\left(m>2 M_{\odot}\right)$ and for brown dwarfs}

A seminal argument suggesting that massive stars form in regions of high density void of low-mass stars but preferentially in tight binaries with similar component masses has been provided by Clarke \& Pringle (1992). They reach this conclusion on the basis of the distribution of OB runaway stars finding these conditions to be necessary. In Bonn, Seungkyung Oh is building on these semi-analytical results by performing direct Nbody calculations of realistic young clusters and testing different pairing rules for massive stars in initially mass-segregated and unsegregated clusters. The general result is that massive stars indeed need high densities and tight binaries with mass-ratios nearby unity in order to account for their spatial distribution around star forming regions.

Brown dwarfs (BDs) form an altogether distinct population from stars as is deduced by Thies \& Kroupa (2008) on the basis of the observed different pairing properties of brown dwarfs and very-low-mass stars on the one hand side, and stars on the other hand side. The most famous evidence for this comes from the brown-dwarf desert: while stars pair up irrespectively of their masses (e.g. G-dwarfs typically have M-dwarf companions), BDs 

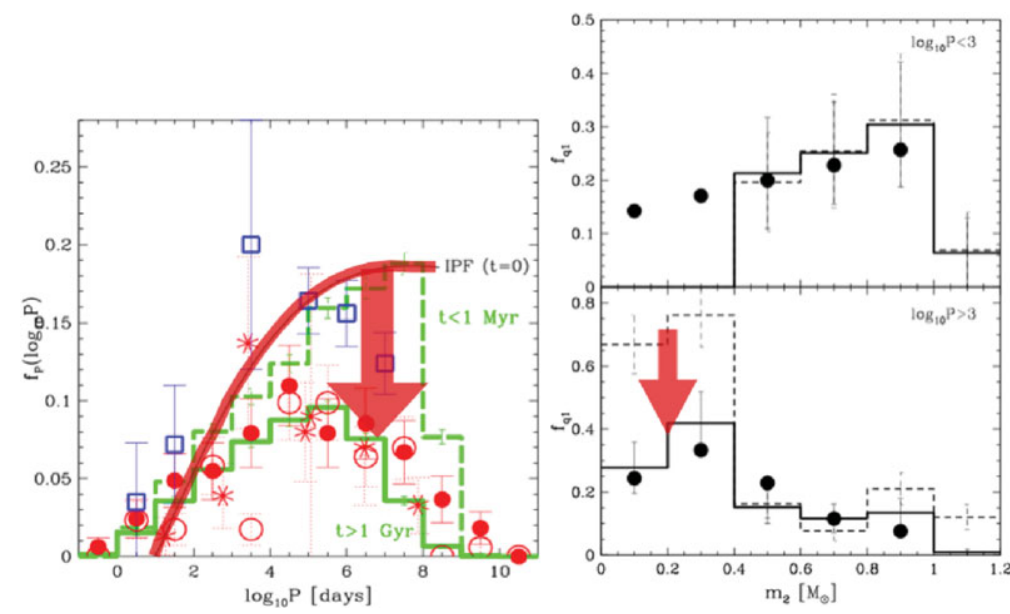

Figure 2. Left panel: The transformation of the birth period distribution function (BPF, Eq. 4.2, thick red curve with $\eta=2.5, \delta=45, l P_{\min }=1$, Kroupa $\left.(1995 \mathrm{~b})=\mathrm{K} 2\right)$ first to the eigenevolved "initial" period distribution function (IPF, dashed green histogram which can be described by Eq. 4.2 with $\eta \approx 3.5, \delta \approx 100, l P_{\mathrm{min}}=0$, Kroupa $\left.(1995 \mathrm{a})=\mathrm{K} 1\right)$. The green solid histogram is the final PF after $\Omega^{130 M_{\odot}, 0.8 \mathrm{pc}}$ acts on the IBP. The solid dots, open circles and stars are G-, K-, and M-dwarf binaries, and the open squares are pre-main sequence systems (see Kroupa (2008) for references). The normalisation of this plot is such that each period bin contains the fraction of binary orbits in the whole sample of stars plus binaries. Right panel: The transformation of the initial mass-ratio distribution for primary masses $m_{1} \approx 1 M_{\odot}$ (dashed histogram) to the final mass-ratio distribution after the population emerges from its star cluster (solid histogram, from K2). The upper panel is for short-period binaries which are not affected by $\Omega^{130 M_{\odot}, 0.8 \mathrm{pc}}$, while the bottom panel shows the long-period binaries. Note that the birth mass-ratio distribution, which results from random pairing from the IMF, is given by the dashed histogram in the lower panel, while eigenevolution transforms this distribution to the dashed histogram evident in the upper panel. That pre-main sequence, i.e. dynamically unevolved, mass-ratios are consistent with random paring from the IMF has been found by Woitas et al. (2001).

are exempt from participating (there are exceptions, but such exceptions are a natural outcome of stellar-dynamical processes). BDs also have a very different semi-major axis distribution, by being limited to $a \lesssim 20 \mathrm{AU}$, while for stars $a$ ranges over five orders of magnitude. Also, BDs have a small binary fraction $f \approx 0.15$ with a bias towards similar companion masses. Computing the effects of the pairing properties of BDs on the observed mass function, Thies \& Kroupa (2008) deduce that the IMF must be discontinuous near $0.08 M_{\odot}$.

Massive stars and BDs thus cannot be included in the BBP formalism above, but require their own mathematical rules. In terms of physics this means that the formation of massive stars and brown dwarfs occur in a physical regime different to that of the typical star.

This is naturally understandable by noting that massive stars can only form in the densest regions of their parent embedded cluster. BDs, on the other hand, typically form in the outer regions of extended circum-stellar discs of the average star, either by disc instability (Goodwin \& Whitworth (2007)) or by tidally-induced gravitational instability caused by passing stars in the parent cluster (Thies et al. (2010)), or are ejected embryos (Reipurth \& Clarke (2001)).

In terms of numbers, massive stars and brown dwarfs are much rarer than the typical star: on average one BD forms per five late-type stars, while one massive star forms per few hundred late-type stars. 

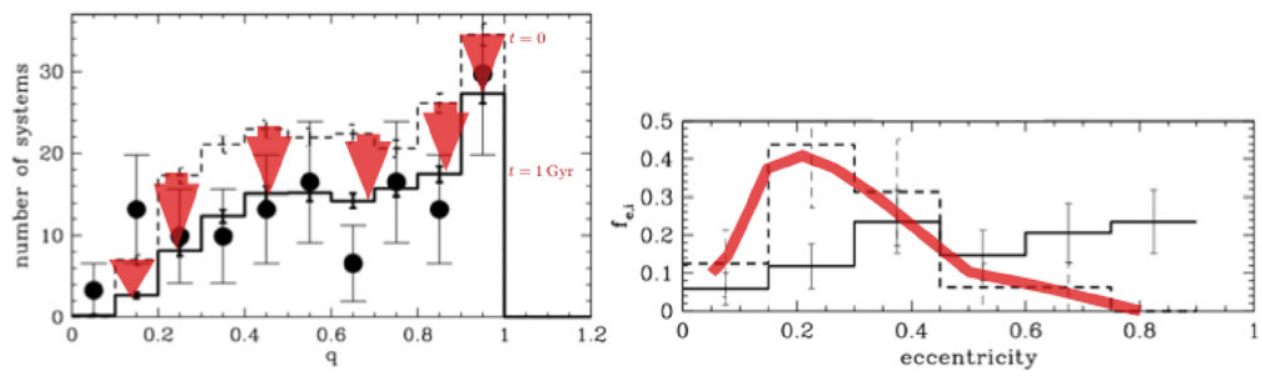

Figure 3. Left panel: The transformation of the overall initial mass-ratio distribution for $0.8<m_{1} / M_{\odot}<1$ (dashed histogram) to the final mass-ratio distribution after the population emerges from its star cluster (solid histogram, from Kroupa (2008)). The $q=1$ peak results from pre-main sequence eigenevolution. The solid circles are observational data by Reid \& Gizis (1997). Right panel: The eccentricity distribution for short-period ( $l P<3$, dashed histogram) and long period $(l P>3$, solid histogram) binaries. The thick red solid curve visualises the bell-shaped distribution of short-period orbits which results from the thermal distribution (the solid histogram) after eigenevolution. Stellar-dynamical encounters, i.e. $\Omega^{M_{\mathrm{ecl}}, R_{0.5}}$ have no effect on the thermal eccentricity distribution (it is an invariant to $\Omega^{M_{\mathrm{ecl}}, R_{0.5}}$ ).

\section{IBP mass regimes and IMF structure}

A possibly interesting issue thus emerges: While the $\mathrm{BD} /$ star schism is clearly evident in the different pairing rules and the discontinuity in the IMF near $0.08 M_{\odot}$, the change of pairing rules between late-type and early-type stars (roughly at a few $M_{\odot}$ ) does not seem to be evident in any corresponding structure in the IMF. In fact, the flattening of the IMF near $0.5 M_{\odot}$ does not seem to correspond to a change in birth binary-star properties. While this fact does not contradict the above statements, it does provide an additional constraint on star-formation theories.

\section{Dynamical Population Synthesis (DyPoS)}

Having thus obtained a formal mathematical description of the invariant birth binary population it now becomes possible to perform Dynamical Population Synthesis (DyPoS). DyPoS rests on the same ansatz as has already been applied to model the vertical structure of the Milky Way disk (Kroupa (2002)) and to model the stellar initial mass function of a whole galaxy (Kroupa \& Weidner (2003)) by adding up the contributions by each embedded cluster. This LEGO PRINCIPLE comes about from the realisation that stars that ultimately end up populating the field form in groups embedded in gas (e.g. Lada \& Lada (2003)).

If the birth stellar population in the groups and the transformations that occur within the groups before the population hatches into the field are understood and are known, then an entire galaxy can be synthesized. This principle is easy to implement for the integrated initial mass function (IGIMF). It is a little bit more involved when implementing it for an integrated description of the vertical structure of the Milky Way disk because the stellar velocity field emerging from an embedded stellar group needs additional assumptions on its time-evolving properties.

Along the same lines, the binary population in a galaxy follows from adding up all the binary populations born in the embedded groups taking into account the dynamical 
transformation that acts on the population in each group as it evolves and disperses,

$$
\begin{aligned}
\mathcal{D}_{\text {field }}\left(l P, e, q: m_{1}\right)= & \int_{M_{\mathrm{ecl}, \mathrm{min}}}^{M_{\mathrm{ecl}, \mathrm{max}}} \int_{R_{0.5, \text { min }}}^{R_{0.5, \text { max }}} \Omega^{M_{\mathrm{ecl}}, R_{0.5}}\left[\Omega_{\text {eigenevol }}\left[\mathcal{D}_{\text {birth }}\left(l P, e, q: m_{1}\right)\right]\right] \\
& \times \xi_{\mathrm{ecl}}\left(M_{\mathrm{ecl}}, R_{0.5}\right) d R_{0.5} d M_{\mathrm{ecl}},
\end{aligned}
$$

where $\xi_{\mathrm{ecl}}\left(M_{\mathrm{ecl}}, R_{0.5}\right)$ is the distribution of stellar masses and half-mass radii of embedded aggregates of stars ("embedded clusters") forming in a time interval $\delta t(\approx 10 \mathrm{Myr})$ throughout the galaxy. Eq. 7.1 sums up all the evolved populations of binaries that are formed in $\xi_{\mathrm{ecl}} d R_{0.5} d M_{\mathrm{ecl}}$ clusters. $R_{0.5} \approx 0.4 \mathrm{pc}$ for embedded clusters and $\xi_{\mathrm{ecl}} \propto M_{\mathrm{ecl}}^{-\beta}$ is the power-law mass function $(\beta=2$ is the usually found index from observational surveys).

Two examples of DyPoS have already been computed by Michael Marks at Bonn University. Eq. 7.1 is solved on a grid of $R_{0.5}$ and power-law index, $\beta$, of the embedded group or cluster initial mass function, $\xi_{\mathrm{ecl}}\left(M_{\mathrm{ecl}}\right)$, where the maximal star-cluster mass, $M_{\text {ecl,max }}$ follows from the star-formation-rate (SFR) versus $M_{\text {ecl,max }}$ relation of Weidner et al. (2004). In an elliptical galaxy which formed in a burst with $S F R=10^{4} M_{\odot} / \mathrm{yr}$ the most-massive star-cluster weighs about $10^{8} M_{\odot}$ corresponding to the mass-scale of ultracompact dwarf galaxies. Binary systems are disrupted efficiently in the massive clusters. Using Eq. 7.1 and assuming the typical embedded star cluster has a radius of $0.4 \mathrm{pc}$, the binary fraction of the late-type stellar population is computed to be $f_{\text {bin }}=0.45$. In a dIrr galaxy with a $S F R=10^{-4} M_{\odot} /$ yr the most massive cluster that can form has a mass of about $M_{\mathrm{ecl} \text {,max }}=100 M_{\odot}$. For $R_{0.5}=0.4 \mathrm{pc}$ DyPoS yields $f_{\mathrm{bin}}=0.85$, because many more wider binaries survive the on average less-massive embedded clusters in the dIrr galaxy, compared to the above star-burst E galaxy.

A full mathematical treatment is found in Marks, Oh \& Kroupa (2010).

\section{Conclusions}

It appears that the star-formation outcome in terms of stellar masses and multiple systems can be formulated by the Star Formation Universality Hypothesis ( $\S 3$ ). For stars with $m \lesssim 2 M_{\odot}$ the BirTh Binary Population $(\S 4)$ can be defined. This is the outcome of star formation in low to intermediate density $\left(\rho \lesssim 10^{4} M_{\odot} / \mathrm{pc}^{3}\right)$ cloud regions (e.g. of an embedded cluster). For $m \gtrsim 2 M_{\odot}$ stars the pairing rules change $(\S 5)$ perhaps reflecting the outcome of star formation in dense regions such as in the cores of embedded clusters $\left(\rho \gtrsim 10^{5} M_{\odot} \mathrm{pc}^{3}\right)$. Brown dwarfs follow entirely separate rules $(\S 5)$ being an accompanying but distinct population to stars. It remains to be understood why these changing IBP properties do not correspond to the structure evident in the $\operatorname{IMF}(\S 6)$.

Acknowledgments: I thank the organizers for a stimulating and memorable conference in Barcelona. My warmest gratitude I wish to express to Sverre Aarseth for his brilliant work on Nbody codes which are freely available and without which this work would not have been possible, and for his unwavering support. This text was written while being a Visitor at ESO/Garching, and I am thankful for the kind hospitality of my colleagues there.

\section{References}

Carney, B. W., Aguilar, L. A., Latham, D. W., \& Laird, J. B. 2005, AJ, 129, 1886

Clarke, C. J. \& Pringle, J. E. 1992, MNRAS, 255, 423

Connelley, M. S., Reipurth, B., \& Tokunaga, A. T. 2008, AJ, 135, 2526 
Duchêne, G. 1999, A\&A, 341, 547

Duquennoy A. \& Mayor M., 1991, A\&A, 248, 485 (DM)

Durisen R.H. \& Sterzik M.F., 1994, A\&A, 286, 84

Fisher, R. T. 2004, ApJ, 600, 769

Goodwin, S. P. \& Whitworth, A. 2007, A\&A, 466, 943

Horton A.J., Bate M.R., \& Bonnell I.A., 2001, MNRAS, 321, 585

Kroupa, P. 1995a, MNRAS, 277, 1491 (K1)

Kroupa, P. 1995b, MNRAS, 277, 1507 (K2)

Kroupa, P. 2002, MNRAS, 330, 707

Kroupa, P. 2008, The Cambridge N-Body Lectures, Lecture Notes in Physics, 760, 181

Kroupa, P. \& Weidner, C. 2003, ApJ, 598, 1076

Lada, C. J. \& Lada, E. A. 2003, ARAA, 41, 57

Marks, M., Oh, S., \& Kroupa, P., 2010, submitted

Moeckel, N. \& Bate, M. R. 2010, MNRAS, 404, 721

Pflamm-Altenburg, J. \& Kroupa, P. 2010, MNRAS, 404, 1564

Reid, I. N. \& Gizis, J. E. 1997, AJ, 113, 2246

Reipurth B., 2000, AJ, 120, 3177

Reipurth, B. \& Clarke, C. 2001, AJ, 122, 432

Sterzik M.F. \& Durisen R.H., 1998, A\&A, 339, 95

Thies, I. \& Kroupa, P. 2008, MNRAS, 390, 1200

Thies, I., Kroupa, P., Goodwin, S. P., Stamatellos, D., \& Whitworth, A. P. 2010, ApJ, 717, 577

Weidner, C., Kroupa, P., \& Larsen, S. S. 2004, MNRAS, 350, 1503

Woitas, J., Leinert, C., Köhler, R. 2001, A\&A, 376, 982 


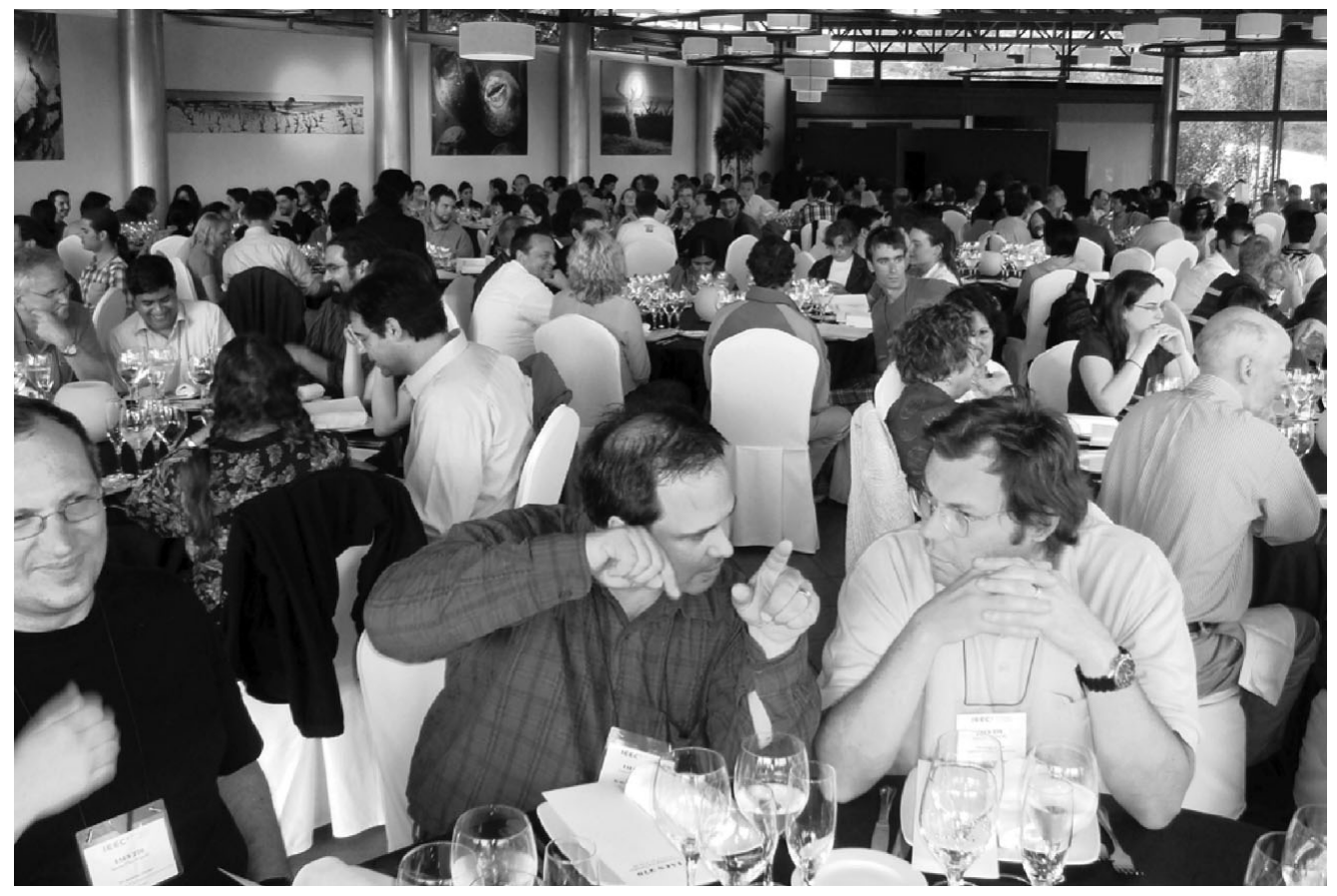

Carsten Weidner, Jan Pflamm-Altenburg, and Pavel Kroupa at the banquet 